\title{
A thorough dynamic interpretation of residual dipolar couplings in ubiquitin
}

\author{
Nils A. Lakomek ${ }^{\mathrm{a}}$, Teresa Carlomagno ${ }^{\mathrm{a}}$, Stefan Becker ${ }^{\mathrm{a}}$, Christian Griesinger $^{\mathrm{a}, *}$ \& \\ Jens Meiler ${ }^{\mathrm{b}, *}$ \\ ${ }^{\mathrm{a}}$ NMR based structural biology, Max Planck Institute for Biophysical Chemistry, Am Fassberg 11, 37077, \\ Goettingen, Germany; ${ }^{\mathrm{b}}$ Center of Structural Biology, Department of Chemistry, Vanderbilt University, 465 \\ 21st Ave South, Nashville, TN, 37232-8725, USA
}

Received 5 September 2005; Accepted 14 November 2005

Key words: dynamical averaging, model free approach, nuclear magnetic resonance (NMR), residual dipolar couplings (rdes), ubiquitin

\begin{abstract}
The presence of slow motions with large amplitudes, as detected by measurements based on residual dipolar couplings [Peti, W., Meiler, J., Brueschweiler, R. and Griesinger, C. (2002) J. Am. Chem. Soc., 124, 58225833], has stirred up much discussion in recent years. Based on ubiquitin NH residual dipolar couplings (rdcs) measured in 31 different alignment conditions, a model-free analysis of structure and dynamics [Meiler, J., Peti, W., Prompers, J., Griesinger, C. and Brueschweiler, R. (2001) J. Am. Chem. Soc., 123, 6098-6107] is presented. Starting from this broad experimental basis, rdc-based order parameters with so far unattained accuracy were determined. These rdc-based order parameters underpin the presence of new modes of motion slower than the inverse overall tumbling correlation time. Amplitudes and anisotropies of the motion were derived. The effect of structural noise on the results was proven to be negligible.
\end{abstract}

\section{Introduction}

Local structural interconversion of proteins, i.e. protein dynamics, is at the center of structural biology when it comes to enzyme function or protein/protein recognition. The existence of motion in the backbone of folded proteins in a time scale shorter than the correlation time $\tau_{\mathrm{c}}$ is widely accepted (Kay et al., 1989; Tjandra et al., 1995). This motion is connected with the libration e.g. of the NH vector (Palmer, 2004). Another type of motion ranges from $100 \mu \mathrm{s}$ to $10 \mathrm{~ms}$ ( $\mu \mathrm{s} / \mathrm{ms}$-motion) and is measured from relaxation dispersion (Kopple et al., 1986; Akke and Palmer, 1996). $\mu \mathrm{s} / \mathrm{ms}$-Motion is invoked when hydrogen bridges open and close and enzymatic reactions occur, which underlines the functional relevance of this time scale (Massi et al.,

*To whom correspondence should be addressed. E-mail: cigr@nmr.mpibpc.mpg.de
2005). However, it has been controversial whether motions occur on a time scale between the rotational correlation time $\tau_{\mathrm{c}}$ and the $\mu \mathrm{s} / \mathrm{ms}$-range in folded proteins and whether they are functionally relevant. While the functional relevance of such motions for the aggregation speed of natively unfolded proteins involved in neurodegenerative diseases has been recently shown (Bertoncini et al., 2005), it is still a matter of debate whether motions on this time scale are present in folded proteins.

The time-window of dynamics that can be characterized by NMR spectroscopy is significantly widened by the measurement of residual dipolar couplings (rdes) (Tolman et al., 1997). Rdcs, which reflect dynamic averaging in the picosecond to millisecond time range, are a unique tool to access important dynamic information especially in the time-window between ca. 4 ns and $50 \mu \mathrm{s}$, which is inaccessible by conventional NMR relaxation methods (Figure 1). 


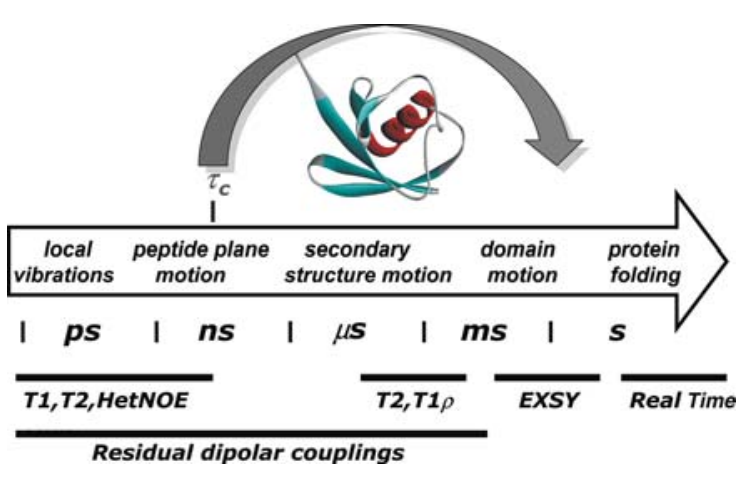

Figure 1. NMR measurements are suitable to detect motion on time scales from sub-ps to hours. Compared to other methods, residual dipolar couplings cover a particular large time range, including the ns to $\mu$ s time range that was so far inaccessible for experimental observation by NMR. The ticks under the arrow should not be considered as upper and lower bounds for the respective motion.

The protein ubiquitin has been the focus of extensive NMR investigations. Besides the determination of a very precise NMR structure (Cornilescu et al., 1998), several ${ }^{15} \mathrm{~N}$ backbone relaxation studies (Schneider et al., 1992; Tjandra et al., 1995; Lienin et al., 1998) and H-D exchange experiments (Johnson et al., 1999; Sivaraman et al., 2001) have been reported. Very recently, an ensemble of conformations has been generated that represents simultaneously the native structure of ubiquitin and its associated dynamics faster than the correlation time $\tau_{\mathrm{c}}$ (Lindorff-Larsen et al., 2005). High pressure NMR spectroscopy revealed two conformations that are in exchange, probably slower than the correlation time (Kitahara et al., 2005).

Having presented two dynamical models to reproduce the experimental rdcs (Tolman et al., 1995; Tolman and Prestegard, 1996a, b) of cyanometmyoglobin, Tolman et al. (1997) proposed an approach to extract dynamics from a set of rdcs measured along the backbone of ubiquitin (Tolman et al., 2001). Seven different heteronuclear rdcs obtained in only one alignment medium were used to derive a general degree of order value (GDO) that reflects the motional scaling of the rdcs for each peptide moiety.

Recently, we proposed a "model-free" approach to the dynamic interpretation of rdcs of a single dipolar vector measured in multiple alignments (Meiler et al., 2001). The model-free approach was compared to a $10 \mathrm{~ns}$ molecular dynamics (MD) simulation of ubquitin and applied to experimental NH rdcs of ubiquitin (Peti et al., 2002). We developed a mathematical framework that allows the extraction of averages of spherical harmonics of rank 2, $\left\langle Y_{2 M}(\theta, \phi)\right\rangle$ for each NH vector. Additionally, by this mathematical analysis we are able to derive effective vector orientations $\left(\theta_{\mathrm{eff}}, \phi_{\mathrm{eff}}\right)$, which define the symmetry axis of the symmetric motion and correspond in good approximation to the average orientations $\left(\theta_{\mathrm{av}}, \phi_{\mathrm{av}}\right)$ extracted from the trajectory of the simulation. The great advantage of the $\left\langle Y_{2 M}(\theta, \phi)\right\rangle$ resides in their property of reflecting motional averages independent of a concrete motional model, in analogy to the model-free approach of Lipari and Szabo (1982a, b) used for the interpretation of NMR spin relaxation data. The $\left\langle Y_{2 M}(\theta, \phi)\right\rangle$ can be used to calculate an $S_{\mathrm{rdc}}^{2}$ order parameter that is similar to the Lipari-Szabo $S_{\mathrm{LS}}^{2}$ order parameter, with the important difference that $S_{\text {rdc }}^{2}$ reflects motions on a time range between femtoseconds and milliseconds while $S_{\mathrm{LS}}^{2}$ represents motions on (sub-) nanosecond time scales. Moreover, the asymmetry of the motional distribution could be characterized by two newly introduced parameters: $\eta_{\text {rdc }}$, which describes the amplitude of the asymmetry, and the angle $\bar{\phi}_{\text {rdc }}^{\prime}$, which defines the direction of anisotropy (Meiler et al., 2003). Application of the model-free approach yields dynamic information on a time window between the ps to low ms time scale.

Tolman developed an independent approach (Tolman, 2001, 2002; Tolman et al., 2001; Briggman and Tolman, 2003), where NH rdcs measured in at least five different alignment media are used to derive the minimum amount of motion that explains the rdcs. Similar to our findings, they observed an overall reduced rdc-derived order parameter compared to the Lipari-Szabo order parameter. In a more recent work Clore and Schwieters described anisotropic motion by a twosite jump model (Clore and Schwieters, 2004), which provides the minimal amount of anisotropic backbone dynamics necessary to be invoked to agree with the experimental rdc data. Finally, the impact of rdcs on understanding slower motion of the peptide plane has been recently described by Bernado and Blackledge (2004a, b).

In this article we present a model-free analysis of residual dipolar couplings in ubiquitin (Meiler et al., 2001; Peti et al., 2002) on the basis of a 
significantly broadened data set which allows us to considerably improve the accuracy of the motional parameters derived in our previous work and thus evaluate the influence of structural noise on the derived apparent motion.

\section{Materials and methods}

\section{Experimental}

Compared to our previous publication (Peti et al., 2002) in which 11 different conditions for alignment were used, the data basis was substantially broadened by performing the following experiments:

A first data set was recorded using five alignment media: DMPC/DHPC bicelles (D1), CHAPSO/DLPC/SDS (D2), n-dodecyl-penta(ethylene glycol)/n-hexanol (D3), purple membrane fragments (D4) and Pf-1 phages (D5). The second set of alignment media included polyacrylamide gel (E1), cetylpyridinium bromide/n-hexanol (E2), n-dodecyl-penta(ethylene glycol)/n-hexanol (E3), purple membrane fragments (E4) and Pf-1 phages (E5).

\section{Sample preparation}

${ }^{15} \mathrm{~N},{ }^{13} \mathrm{C}$-labeled human ubiquitin (wt) was purchased from VLI Research, Inc. (Malvern, PA) and used without further purification for the data sets D1, D2, D3, D4, D5, E1 and E4. For the data sets E2, E3 and E5 we used ${ }^{15} \mathrm{~N},{ }^{13} \mathrm{C}$-labeled human ubiquitin produced according to Johnson et al. (1999). Both ubiquitin samples gave identical HSQC-spectra (vide infra). To estimate the strength of alignment, the water ${ }^{2} \mathrm{H}$ quadrupolar splitting (QS) has been measured for each alignment medium.

Preparation of the first set. For the first set of alignment media, all samples contained $0.4-0.6 \mathrm{mM}$ of ${ }^{15} \mathrm{~N},{ }^{13} \mathrm{C}$-labeled human ubiquitin in $10 \mathrm{mM} \mathrm{Na}$ phosphate buffer, $\mathrm{pH}=6.5$, including $10 \%$ of $\mathrm{D}_{2} \mathrm{O}$. Two bicelle media, DMPC:DHPC $=3: 1(5 \% \mathrm{w} / \mathrm{v}$, Avanti Polar Lipids, sample D1) (Cornilescu et al., 1998) and CHAPSO:DLPC:SDS = 125:25:1 (5\% w/v, Avanti Polar Lipids, sample D2) (Losonczi and Prestegard, 1998) were prepared. Sample D3 contained dodecyl-penta(ethylene glycol) (C12E5) and $n$-hexanol in $10 \mathrm{mM} \mathrm{Na}$ phosphate buffer, $\mathrm{pH}=6.5$. The $\mathrm{C} 12 \mathrm{E} 5$ surfactant-to-water ratio was $5 \%(\mathrm{w} / \mathrm{w})$ and the molar ratio of surfactant/alco- hol was 0.985 (Ruckert and Otting, 2000). Sample D4 was prepared by adding purple membrane fragments at $4 \mathrm{mg} / \mathrm{ml}$ to the protein solution. Additionally, $100 \mathrm{mM} \mathrm{NaCl}$ (final concentration) was added to reduce electrostatic interactions between purple membrane and ubiquitin (Koenig et al., 1999). For the fifth alignment condition (sample D5), $17 \mathrm{mg} / \mathrm{ml} \mathrm{Pf-1} \mathrm{Phages} \mathrm{(ASLA} \mathrm{Ltd.,}$ Riga, Latvia) were dissolved in $10 \mathrm{mM}$ Na phosphate buffer, $\mathrm{pH}=6.5$. Additionally, $\mathrm{NaCl}$ was added up to a final concentration of $350 \mathrm{mM}$ in order to reduce electrostatic interactions between phages and ubiquitin (Zweckstetter and Bax, 2001). A $0.4 \mathrm{mM}$ isotropic solution of ${ }^{15} \mathrm{~N},{ }^{13} \mathrm{C}$ labeled human ubiquitin was used for reference (D6).

Preparation of the second set. For all samples of the second set of alignment media the concentration of ${ }^{15} \mathrm{~N},{ }^{13} \mathrm{C}$-labeled human ubiquitin was increased to $0.6-1.1 \mathrm{mM}$. The $\mathrm{Na}$ phosphate buffer, $\mathrm{pH}=6.5$, was increased from 10 to $50 \mathrm{mM}$ and $10-15 \% \quad \mathrm{D}_{2} \mathrm{O}$ was added. Six samples, termed E1-E6, were prepared. In sample E1 the protein solution was soaked into a $7 \%$ uncharged polyacrylamide gel. In these gels alignment is achieved by radially compressing and stretching the gel in the direction of the magnetic field (Sass et al., 2000; Chou et al., 2001). Alignment in sample E2 was achieved by preparing a $3.5 \%(\mathrm{w} / \mathrm{v})$ solution of cetylpyridinium bromide and $n$-hexanol in $50 \mathrm{mM}$ Na phosphate buffer, $\mathrm{pH}=6.5$, and $25 \mathrm{mM}$ $\mathrm{NaBr}$. The molar ratio of $\mathrm{CPBr}$ and $n$-hexanol was 1:1 (Barrientos et al., 2000). Sample E3 was very similar to sample D3, except for the $\mathrm{Na}$ phosphate buffer concentration, which was $50 \mathrm{mM}$ instead of $10 \mathrm{mM}$. Sample E4 was prepared similar to sample D4 except increasing the concentration of the $\mathrm{Na}$ phosphate buffer from 10 to $50 \mathrm{mM}$ and decreasing the concentration of $\mathrm{NaCl}$ from 100 to $50 \mathrm{mM}$. For sample E5 the Pf-1 Phage concentration was reduced to $15 \mathrm{mg} / \mathrm{ml}$ (compared to $17 \mathrm{mg} / \mathrm{ml}$ in sample D5), whereas the $\mathrm{NaCl}$ concentration was increased from 350 to $400 \mathrm{mM}$. For reference a $50 \mathrm{mM} \mathrm{Na}$ phosphate buffer $(\mathrm{pH}=6.5)$ with $1.8 \mathrm{mM}{ }^{15} \mathrm{~N},{ }^{13} \mathrm{C}$-labeled ubiquitin was used as isotropic solution (Sample E6).

\section{NMR Spectroscopy}

NMR experiments for the first dataset were performed on a Bruker-avance $700 \mathrm{MHz}$ spectrome- 
ter (Bruker AG, Karlsruhe, Germany) at 308 K. A two dimensional ${ }^{15} \mathrm{~N},{ }^{1} \mathrm{H}-\mathrm{HSQC}$ experiment was run to measure $\mathrm{NH}$ rdcs. The time domain was $\mathrm{TD} 1 \times \mathrm{TD} 2=1 \mathrm{k} \times 2 \mathrm{k}$. For the second data set all NMR experiments were performed on BrukerDRX-600 MHz and Bruker-avance- $800 \mathrm{MHz}$ spectrometers (Bruker AG, Karlsruhe, Germany).

To measure the NH rdcs of ubiquitin in the different alignment media 2D-IPAP- ${ }^{15} \mathrm{~N},{ }^{1} \mathrm{H}$ - HSQC experiments (Ottiger et al., 1998) were recorded for each aligned sample (D1-D5 and E1-E5) as well as for the isotropic sample (D6 and E6). All IPAP spectra were recorded in the interleaved mode at $308 \mathrm{~K}$. Temperatures were calibrated by using 1,2ethanediol. The temperature-dependent chemical shift difference between the $\mathrm{OH}$ protons and those of the methylene groups was measured.

For the Bruker-DRX-600 MHz spectra the time domain wasTD $1 \times$ TD2 $=512 \times 2048$ complexpoints, the spectral width $\mathrm{F} 1 \times \mathrm{F} 2=1818 \mathrm{~Hz} \times 8389 \mathrm{~Hz}$, $\mathrm{NS}=48$ and the total experimental time $25 \mathrm{~h}$, for the Bruker-avance- $800 \mathrm{MHz}$ spectra the time domain was $\mathrm{TD} 1 \times \mathrm{TD} 2=768 \times 2048$, the spectral width $\mathrm{F} 1 \times \mathrm{F} 2=2432 \mathrm{~Hz} \times 11160 \mathrm{~Hz}, \mathrm{NS}=32$ and the total experimental time $20 \mathrm{~h}$. After zero-filling to $\mathrm{TD} 1 \times \mathrm{TD} 2=32 \mathrm{k} \times 4 \mathrm{k}$ and processing the spectra the couplings were extracted using the NMRPipe software package (Delaglio et al., 1995). Alignment tensors were calculated using the DipoCoup software (Meiler et al., 2000). One bond ${ }^{15} \mathrm{~N}-{ }^{1} \mathrm{H}$ rdcs were derived from the difference in splitting between the aligned samples (1-5) and the isotropic state (6). A conservative estimate for the experimental error is $0.3 \mathrm{~Hz}$ for the measured $\mathrm{NH}$ rdc, since the digitalization is $0.055 \mathrm{~Hz} /$ point after zero-filling.

As shown in Figure 2, the correlation of measured rdcs versus back-calculated values is excellent. Using the NMR-structure 1d3z (Cornilescu et al., 1998) for back-calculation the correlation coefficient is $\rho \geq 0.99$, the $Q$-value varies between 0.10 for polyacrylamide gel and 0.14 for cetylpyridinium bromide $/ n$-hexanol. When the $\mathrm{X}$-ray structures 1ubi (Ramage et al., 1994), 1ubq (Vijay Kumar et al., 1987) are used for back-calculation, the correlation decreases only slightly, while the maximum $Q$-value increases to 0.19 .

\section{Input for analysis}

For the first analysis (Peti et al., 2002) 11 sets of NH rdcs were used (A). Nine more data sets were published by Tolman and coworkers (Tolman, 2002) (B) and one dataset, obtained in polyacrylamide gel, was taken from the literature (Sass et al., 2000; Tycko et al., 2000) (C). These data sets were supplemented with the measurements described in the experimental section yielding two sets of five different alignment conditions (D and E). Altogether, a total of 31 experimental datasets were available for the analysis (compare Table 1). By using a large number of experimental data sets as input for the analysis, we could reduce the experimental noise and improve the accuracy of the inversion of the $\hat{F}$ matrix (vide infra). This procedure resulted in very accurate rdc-derived order parameters. Even if some alignment conditions are very similar we find it desirable to use as much rdcdatasets as available to ensure the convergence of the order parameters.

\section{Theory}

The theory of dynamically averaged rdcs has been described in detail in an earlier publication (Meiler et al., 2001). The most essential equations are summarized in the following paragraphs.

\section{Motional averaging of residual dipolar couplings}

The size of the residual dipolar coupling for residue $j$ observed in an alignment medium $i$ is given by

$$
\begin{aligned}
D_{i j}^{\mathrm{exp}}= & \frac{1}{2} D_{i, z z}\left\{\left\langle 3 \cos ^{2} \theta_{i j}^{\mathrm{at}}-1\right\rangle\right. \\
& \left.+\frac{3}{2} R_{i}\left\langle\sin ^{2} \theta_{i j}^{\mathrm{at}} \cos 2 \phi_{i j}^{\mathrm{at}}\right\rangle\right\}
\end{aligned}
$$

The angular brackets denote conformational averaging. $D_{i, z z}$ is the principal component and the rhombicity $R_{i}$ of the alignment tensor induced by the alignment medium $i$. The orientation of the internuclear $\mathrm{NH}$ vector of residue $j$ with respect to the frame of the alignment tensor (at) is described by the polar angles $\theta_{i j}^{\text {at }}$ and $\phi_{i j}^{\text {at }}$. Note, that the following equations assume that the average orientation as well as the dynamics of each $\mathrm{NH}$ vector are independent of the alignment medium and are not correlated with the alignment tensor of the whole molecule. We assume that the ubiquitin structure and the 

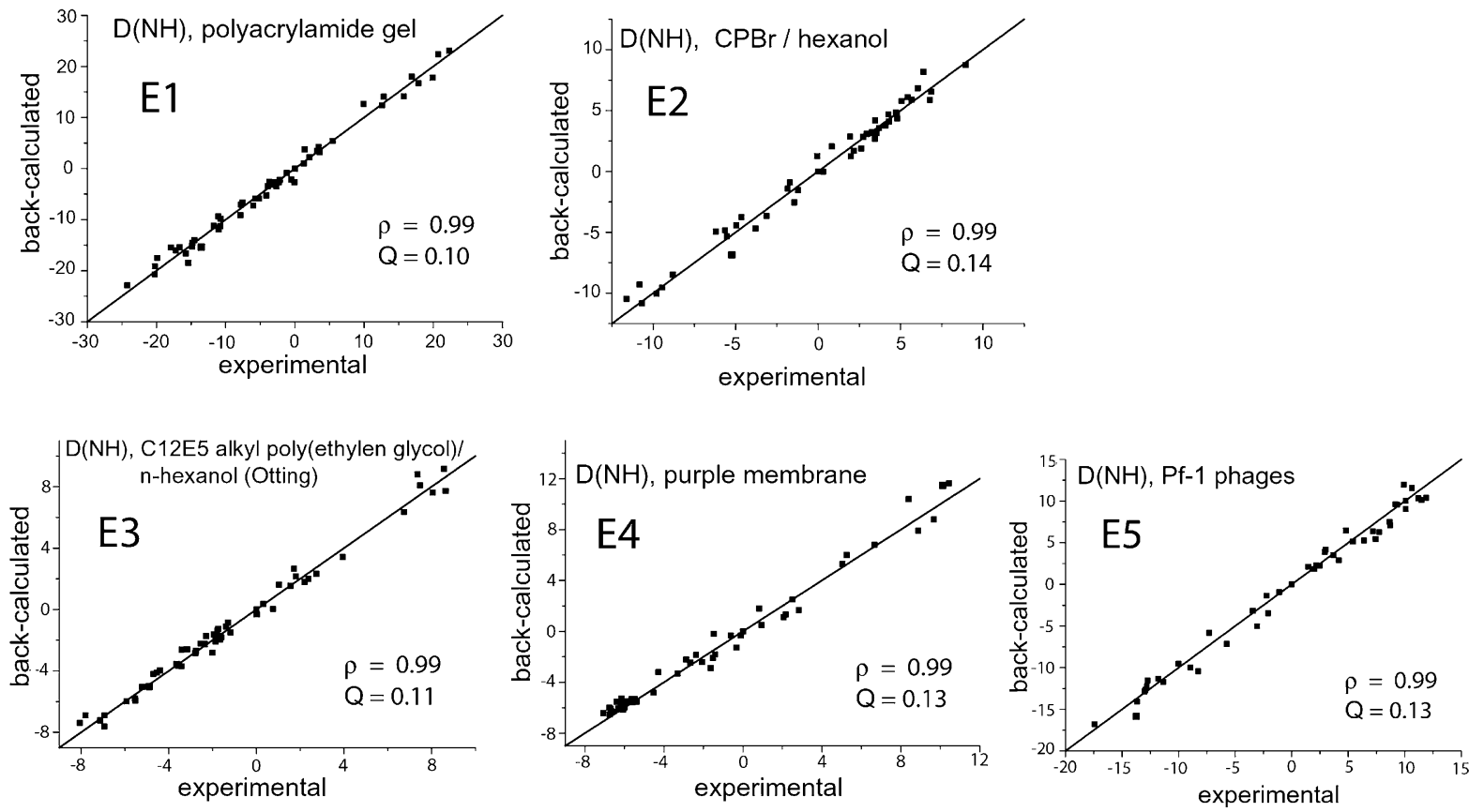

Figure 2. Correlation of experimental residual dipolar couplings with values that were back-calculated from a single static structure $(1 \mathrm{~d} 3 \mathrm{z})$ for a set of five different alignment media. An excellent agreement between a single structural model and the data is already observed $(Q$-values between 0.10 and 0.14$)$. However, the small deviations are not only experimental uncertainty but also reflect dynamics. The alignment media shown are polyacrylamide gel (E1), cetylpyridinium bromide/ $n$-hexanol (E2), $n$-dodecyl-penta(ethylene glycol)/ $n$-hexanol (E3), purple membrane fragments (E4), and Pf-1 phages (E5).

Table 1. Influence of the number of included data set on the eigenvalues of the $\hat{F}$-matrix and the resulting standard deviation for $S_{\text {rdc }}$

\begin{tabular}{lllllllll}
\hline Number of alignments & Number of AA & \multicolumn{3}{c}{ Eigenvalues of $\hat{F}$-matrix } & & $\Delta\left(S_{\text {rdc }}\right)$ & Condition number \\
\hline 7 (subset of A) & 53 & 3.79 & 1.84 & 1.02 & 0.65 & 0.46 & 0.080 & 8.32 \\
11 (A) & 46 & 5.20 & 2.27 & 0.91 & 0.68 & 0.59 & 0.080 & 8.85 \\
17 (A+C+D) & 63 & 6.07 & 2.73 & 1.36 & 0.92 & 0.81 & 0.039 & 7.49 \\
22 (A+C+D+E) & 64 & 6.77 & 3.34 & 1.46 & 1.07 & 0.98 & 0.026 & 6.90 \\
31 (A+B + C + D + E) & 65 & 8.03 & 4.46 & 1.63 & 1.37 & 1.17 & 0.021 & 6.88 \\
\hline
\end{tabular}

$\mathrm{AA}$ is amino acid.

internal dynamics are not affected by the alignment process. Since only slight chemical shift differences for the ubiquitin peaks occur in the spectra for the different alignment media and because it is possible to fit the rdcs to a single dynamically averaged alignment tensor, the mentioned assumptions appear reasonable to us. However, this assumption cannot be made for multi-domain proteins and, therefore, this analysis can only be applied to globular proteins. With this in mind, it is useful to express Equation (1.1) using normalized second-order spherical harmonic functions described in an alignment-independent molecular frame:

$$
\frac{D_{i j}^{\exp }}{D_{i, z z}}=\sum_{M=-2}^{2} F_{i, M}\left\langle Y_{2, M}\left(\theta_{j}^{\mathrm{mol}}, \phi_{j}^{\mathrm{mol}}\right)\right\rangle
$$

where the $\left\langle Y_{2, M}\left(\theta_{j}^{\mathrm{mol}}, \phi_{j}^{\mathrm{mol}}\right)\right\rangle$ are the averaged spherical harmonics for a given $\mathrm{NH}$ vector $j$. The superscript mol in Equation (1.2) indicates that the spherical harmonics refer to the molecular frame. The $F_{i, M}$ terms consist of Wigner-Seitz-Rotation elements (cf. Meiler et al., 2001) and are functions 
of the three Euler angles, $\alpha_{i}, \beta_{i}$ and $\gamma_{i}$, describing the rotation of the molecular frame axes into the alignment frame axes of alignment medium $i$.

Since all measured rdcs are intrinsically scaled by motional averaging, their overall fitting to a single rigid NMR structure necessarily yields a motional averaged alignment tensor characterized by $\tilde{D}_{i, z z}$ and $\tilde{R}_{i}$ as well as the angles $\tilde{\alpha}_{i}, \tilde{\beta}_{i}$, and $\tilde{\gamma}_{i}$. However, for the rdc-based model-free analysis of dynamics a static alignment tensor has to be invoked because all dynamics are represented by the time-averaged spherical harmonics by definition. That is why the static alignment tensor (no tilde) has to be estimated from the experimentally determined dynamically averaged one (indicated by tilde). As has been pointed out in (Meiler et al., $2001)$, the orientation $\left(\tilde{\alpha}_{i}, \tilde{\beta}_{i}, \tilde{\gamma}_{i}\right)$ and the rhombicity $\left(\tilde{R}_{i}\right)$ of the scaled tensor are virtually indistinguishable from those of the true tensor and that the motion is reflected only in a scaling of the principal value according to $\tilde{D}_{i, z z}=S_{\text {overall }} \cdot D_{i, z z}$. We can thus rewrite Equation (1.2):

$$
\frac{D_{i j}^{\exp }}{\tilde{D}_{i, z z}} \cdot S_{\text {overall }}=\sum_{M=-2}^{2} F_{i, M}\left\langle Y_{2, M}\left(\theta_{j}^{\mathrm{mol}}, \phi_{j}^{\mathrm{mol}}\right)\right\rangle
$$

This scaling leads to scaled spherical harmonics $\left\langle Y_{2 M}(\theta, \phi)\right\rangle_{\text {(scaled) }}=S_{\text {overall }} \cdot\left\langle Y_{2 M}(\theta, \phi)\right\rangle_{\text {(unscaled) }}$ since the spherical harmonics are determined by inverting Equation (1.3). It should be noted that it is impossible to obtain the value of $S_{\text {overall }}$ directly from the rdcs. However, a reasonable estimate can be obtained as will be discussed in the paragraph Estimation of $S_{\text {overall }}$.

For the following, the superscript mol will be omitted: $\theta$ and $\phi$ will be used when referring to the molecular frame of the protein.

\section{Model free approach}

The inversion of the $\hat{F}$ matrix in Equation (1.3) yields the averages of the spherical harmonics. Since conjugation commutes with averaging, then the equivalences $\left\langle Y_{22}(\theta, \varphi)\right\rangle^{*} \stackrel{!}{=}\left\langle Y_{2-2}(\theta, \varphi)\right\rangle$ and $\left\langle Y_{21}(\theta, \varphi)\right\rangle^{*} \stackrel{!}{=}-\left\langle Y_{2-1}(\theta, \varphi)\right\rangle$ are valid in Equation (1.3), and only five independent variables remain. Therefore, at least five different alignments have to be experimentally observed in order to determine the five spherical harmonic terms $\left\langle Y_{2 M}(\theta, \phi)\right\rangle$.
From these, an rdc-based order parameter $S_{\text {rdc }}^{2}$ can be determined characterizing the amount of motion present from the picosecond to the low millisecond time range. The rdc-based order parameter $S_{\mathrm{rdc}}^{2}$ is defined analogously to the Lipari-Szabo order parameter $S_{\mathrm{LS}}^{2}$ :

$$
\begin{array}{r}
S_{\mathrm{rdc}}^{2}=\left.\left.\frac{4 \pi}{5} \sum_{M=-2}^{2}\left\langle Y_{2, M}(\theta, \phi)\right\rangle\right|_{\mathrm{ps}} ^{\mathrm{ms}}\left\langle Y_{2, M}^{*}(\theta, \phi)\right\rangle\right|_{\mathrm{ps}} ^{\mathrm{ms}} \\
S_{\mathrm{LS}}^{2}=\left.\left.\frac{4 \pi}{5} \sum_{M=-2}^{2}\left\langle Y_{2, M}(\theta, \phi)\right\rangle\right|_{\mathrm{ps}} ^{\tau_{\mathrm{c}}}\left\langle Y_{2, M}^{*}(\theta, \phi)\right\rangle\right|_{\mathrm{ps}} ^{\tau_{\mathrm{c}}}
\end{array}
$$

The difference between the definitions of the two order parameters lies in the time range over which averaging of the internal motion occurs (cf. Equation (1.4)). Unlike $S_{\mathrm{LS}}^{2}, S_{\text {rdc }}^{2}$ reflects motion up to the time scale that is defined by the inverse of the differences of chemical shifts of rdcs of exchanging conformations (normally in the $\mathrm{ms}$ range). Therefore, the most conservative approach is to impose the condition $S_{\mathrm{LS}}^{2} \geq S_{\mathrm{rdc}}^{2}$, which takes into account all motions contained in the Lipari-Szabo order parameter, such as vibration motions. The rdc-derived order parameter must always be smaller than the Lipari-Szabo derived one as the former picks up motion on all time scales up to the ms range while the latter does not.

An upper limit for the scaling factor $S_{\text {overall }}$ in Equation (1.3) has to be estimated by requiring $S_{\mathrm{LS}}^{2} \geq S_{\mathrm{rdc}}^{2}$ for every individual amino acid in ubiquitin (Peti et al., 2002) (vide infra). If the $S_{\text {overall }}$ scaling was neglected in the above set of equations the derived $\left\langle Y_{2, M}(\theta, \phi)\right\rangle_{\text {unscaled }}=\left\langle Y_{2, M}(\theta, \phi)\right\rangle /$ $S_{\text {overall }}$ and $S_{\text {rdc(unscaled) }}=S_{\text {rdc(scaled) }} / S_{\text {overall }}$ would be increased by a factor $1 / S_{\text {overall }}$. We will refer to these values as "unscaled". The scaling factor can be estimated by analyzing the distribution of $S_{\mathrm{LS}}^{2} / S_{\text {rdc(unscaled) }}^{2}$ values (vide infra). It should be noted that it is intrinsically impossible to derive the value of $S_{\text {overall }}$ directly from the rdcs.

Besides allowing the extraction of $S_{\text {rdc }}^{2}$ in a model-free way, the $\left\langle Y_{2, M}(\theta, \phi)\right\rangle$ values provide a detailed picture of the on-going motional averaging. First, for each individual $\mathrm{NH}$ vector the coordinate system is transformed by a Wigner- 
Rotation into a new (primed) frame such that $\left\langle Y_{2,0}\left(\theta^{\prime}, \phi^{\prime}\right)\right\rangle$ is maximized

$$
\begin{aligned}
\max \stackrel{!}{=}\left\langle Y_{2,0}\left(\theta^{\prime}, \phi^{\prime}\right)\right\rangle & =\sum_{M=-2}^{2} D_{M, 0}\left(\phi_{\mathrm{eff}}, \theta_{\mathrm{eff}}, 0\right) \\
& \left\langle Y_{2, M}(\theta, \phi)\right\rangle \\
& =\sqrt{\frac{4 \pi}{5}} \sum_{M=-2}^{2} Y_{2 M}^{*}\left(\theta_{\mathrm{eff}}, \phi_{\mathrm{eff}}\right) \\
& \left\langle Y_{2, M}(\theta, \phi)\right\rangle
\end{aligned}
$$

Maximizing $\left\langle Y_{2,0}\left(\theta^{\prime}, \phi^{\prime}\right)\right\rangle$ places the new $z$ axis into the center of the distribution for the given $\mathrm{NH}$ vector and thus defines the polar angles $\theta_{\text {eff }}$ and $\phi_{\text {eff }}$, and results in $\left\langle Y_{2,1}\left(\theta^{\prime}, \phi^{\prime}\right)\right\rangle$ and $\left\langle Y_{2,-1}\left(\theta^{\prime}, \phi^{\prime}\right)\right\rangle$ becoming equal to zero. $\left\langle Y_{2,2}\left(\theta^{\prime}, \phi^{\prime}\right)\right\rangle$ and $\left\langle Y_{2,-2}\left(\theta^{\prime}, \phi^{\prime}\right)\right\rangle$ represent the asymmetry of the motion. To obtain a better understanding of the asymmetric part of motion the amplitude $\eta$ of anisotropic motion is introduced

$$
\eta_{\mathrm{rdc}}=\sqrt{\frac{\sum_{M=-2,2}\left\langle Y_{2, M}\left(\theta^{\prime}, \phi^{\prime}\right)\right\rangle\left\langle Y_{2,-M}\left(\theta^{\prime}, \phi^{\prime}\right)\right\rangle}{\sum_{M=-2,0,2}\left\langle Y_{2, M}\left(\theta^{\prime}, \phi^{\prime}\right)\right\rangle\left\langle Y_{2,-M}\left(\theta^{\prime}, \phi^{\prime}\right)\right\rangle}} .
$$

The direction of the anisotropic motion is described by the angle $\bar{\phi}_{\text {rdc }}^{\prime}$ with respect to the $x^{\prime}$ axis in the $x^{\prime} y^{\prime}$ plane of the primed frame. This angle $\bar{\phi}_{\text {rdc }}^{\prime}$ can be determined by application of an Euler rotation $R\left(\phi_{\mathrm{eff}}, \theta_{\mathrm{eff}}, \bar{\phi}_{\mathrm{rdc}}^{\prime}\right)$ that transforms the molecular frame axes into the axes of a (doubly primed) frame, so that the second order spherical harmonic $Y_{2,2}\left(\theta^{\prime \prime}, \phi^{\prime \prime}\right)$ becomes maximized in that double primed frame

$$
\max \stackrel{!}{=} Y_{22}\left(\theta^{\prime \prime}, \phi^{\prime \prime}\right)=R\left(\phi_{\mathrm{eff}}, \theta_{\mathrm{eff}}, \bar{\phi}_{\mathrm{rdc}}^{\prime}\right) Y_{22}(\theta, \phi) .
$$

Furthermore, the $\phi^{\prime}$ dependence of $\left\langle Y_{2,2}\left(\theta^{\prime}, \phi^{\prime}\right)\right\rangle$ and $\left\langle Y_{2,-2}\left(\theta^{\prime}, \phi^{\prime}\right)\right\rangle$ results in a $\pi$ periodicity of $\bar{\phi}_{\text {rdc }}^{\prime}$. The translation of the five averaged spherical harmonics into the primed coordinate system amounts to the definition of five new parameters, namely: $\left\langle Y_{2,0}\left(\theta^{\prime}, \phi^{\prime}\right)\right\rangle$ which reflects the axial order, $\theta_{\text {eff }}$ and $\phi_{\text {eff }}$ which repre- sent the average orientation of the vector, and $\eta_{\text {rdc }}$ and $\bar{\phi}_{\text {rdc }}^{\prime}$ which reflect the amount of anisotropic disorder and the direction of this anisotropic motion in the $x^{\prime} y^{\prime}$ plane. Note that $\theta_{\mathrm{eff}}, \phi_{\mathrm{eff}}, \eta_{\mathrm{rdc}}$, and ${\overline{\phi^{\prime}}}_{\text {rdc }}^{\prime}$ are not affected by the scaling procedure and therefore also not sensitive to errors in estimating $S_{\text {overall }}$.

In order to visualize the direction of anisotropy more easily, the angle $\xi_{\text {rdc }}^{\prime}$ is defined as the direction of the $\mathrm{NC}_{\alpha}$ vector in the $x^{\prime \prime}, y^{\prime \prime}$ plane (compare Figure S1):

$$
\xi_{\text {rdc }}^{\prime}=\arctan \frac{y_{\mathrm{NC}_{\alpha}}^{\prime \prime}}{x_{\mathrm{NC}_{\alpha}}^{\prime \prime}}
$$

where $y_{\mathrm{NC}}^{\prime \prime}$ and $x_{\mathrm{NC}}^{\prime \prime}$ are the coordinates of the $\mathrm{NC}_{\alpha}$ vector in the doubly primed frame.

The angle $\xi_{\text {rdc }}^{\prime}$ can be regarded as the direction of the anisotropy with respect to the peptide plane. In contrast to $\bar{\phi}_{\text {rdc }}^{\prime}, \xi_{\text {rdc }}^{\prime}$ is no longer model-free, since $\xi_{\text {rdc }}^{\prime}$ is calculated using the NMR ubiquitin structure $(1 \mathrm{~d} 3 z)$ for the definition of the peptide plane.

\section{Quality measures and error assessment}

Out of the original 11 datasets used in our previous publication (Peti et al., 2002), four (CHAPSO/ DLPC, CHAPSO/DLPC/SDS, purple membrane, and polyacrylamide gel) were not used for the new analysis (leaving a total of 27 data sets), as more accurate measurements have since become available in these cases. In addition, the rather large $Q$-values for these datasets suggested relatively high experimental uncertainties.

To identify rdc data affected by high experimental noise, the complete matrix of all rdcs for all 76 amino acids in all 27 alignment media was analyzed with singular value decomposition, as proposed by Tolman (2002). In this analysis the first five eigenvalues represent the five structural as well as dynamical degrees of freedom (compare above), while all other eigenvalues reflect noise. A matrix of rdcs was back-calculated using only the largest five eigenvalues and respective eigenvectors. Those rdc values that deviated by more than $12 \%$ of the absolute value of $D_{z z}$ (three times the standard deviation) from the back-calculated ones were not considered further. About $3 \%$ of all data points were removed by this procedure. 
More than 1400 measured NH couplings have been used for the analysis. To evaluate the influence of each single data set on our analysis each of the 27 remaining data sets was consecutively removed and the analysis was performed without that dataset.

This has been done using tensors derived from three different structures, two X-ray (PDB codes 1ubi, 1ubq) as well as one NMR structure (PDB code $1 \mathrm{~d} 3 \mathrm{z}$ ) to evaluate the influence of structural noise on the analysis. Finally the average and standard deviations of all $27 \times 3=81$ cycles was taken as results for the model free parameters. All protocols used for this analysis were implemented with the MATHEMATICA5 software package.

\section{Results and Discussion}

\section{Number of datasets}

The results of our previous analysis (Peti et al., 2002) suggested, that the number of available alignment media critically influences the quality of the structural and dynamical parameters derived. Although in theory a set of five well-chosen alignment media is sufficient to determine the two structural parameters as well as the three dynamical parameters, the accuracy of the latter in particular improves significantly using additional experimental datasets. This is due to the small size of the changes caused by motional averaging, which approaches the range of experimental uncertainties. Besides the reduction of experimental noise, an efficient way to separate this noise from real dynamical information resides in the improvement of the statistics obtained by the measurement of additional experimental data.

\section{Estimation of $S_{\text {overall }}$}

In order to calibrate $S_{\text {overall, we assume that the }}$ most rigid backbone amide groups with the highest $S_{\text {rdc(unscaled) }}$ experience no additional motion beyond the correlation time $\tau_{\mathrm{c}}$. For those residues holds: $\quad S_{\mathrm{LS}}^{2}=S_{\text {rdc(scaled) }}^{2}=S_{\text {overall }}^{2} S_{\text {rdc(unscaled) }}^{2} . \quad W e$ require $S_{i, \mathrm{LS}}^{2} \geq S_{i, \mathrm{rdc}(\text { scaled) }}^{2}=S_{\text {overall }}^{2} S_{i, \mathrm{rdc} \text { (unscaled) }}^{2}$ or $S_{i, \mathrm{LS}}^{2} / S_{i, \mathrm{rdc} \text { (unscaled) }}^{2} \geq S_{\text {overall }}^{2}$ for all amino acids. Taking the minimum of all $S_{i, \mathrm{LS}}^{2} / S_{i, \mathrm{rdc} \text { (unscaled) }}^{2}$-ratios as $S_{\text {overall }}^{2}$ value which is $S_{\text {overall }}^{2}=0.59$ would ensure that this inequality is always fulfilled. In order to have a statistically more significant basis, we made a histogram analysis of the distribution of $S_{i, \mathrm{LS}}^{2} / S_{i, \mathrm{rdc}(\text { unscaled) }}^{2}$-rations (cf. Figure 3 ) and added standard deviation (0.1) of this distribution to the value of $S_{\text {overall }}^{2}=0.59$ and arrived at $S_{\text {overall }}^{2}=0.69\left(S_{\text {overall }}=0.83\right)$ as a very conservative upper limit for $S_{\text {overall }}^{2}$. Note, that the exact shape of the distribution is not relevant for this conclusion. Note also, that the $S_{\text {overall }}^{2}=0.59\left(S_{\text {overall }}=0.77\right)$ value is very close to the one found in the original paper by Peti et al. 2002 that was $\left(S_{\text {overall }}=0.78\right)$.

Both results, the very conservative $S_{\text {overall }}=0.83$ and the less conservative value $S_{\text {overall }}=0.77$ are still considerably smaller than the Lipari-Szabo averaged order parameter $\overline{S_{\mathrm{LS}}}=0.88$ obtained for $35^{\circ} \mathrm{C}$ (Chang and Tjandra, 2005). In the current analysis all rdc-based order parameters $S_{\mathrm{rdc}}^{2}$ are (within the error bars) smaller than the LipariSzabo counter part $S_{\mathrm{LS}}^{2}$, with a few exceptions for Leu8, Asp32, Gln49 and Ser57 that can be explained by the very conservative estimate for $S_{\text {overall }}$.

\section{Model free parameters}

In Figure 4 the effective orientation of each $\mathrm{NH}$ vector $\left(\theta_{\text {eff }}, \phi_{\text {eff }}\right)$ is compared with the $\mathrm{NH}$ vector orientation found in the ubiquitin structure determined by NMR spectroscopy (PDB code: $1 \mathrm{~d} 3 \mathrm{z})$. All deviations are smaller than $10^{\circ}$ except for some $\phi$ angles when $\theta$ is close to $0^{\circ}$ or $180^{\circ}$. In these cases, $\phi$ becomes undefined since the vector is almost parallel with the $\pm z$ axis of the coordinate frame. Therefore, for the deviations of $\phi_{\mathrm{eff}}$ from the $\phi$ value of the NMR structure we calculated $\Delta \phi_{\text {eff }} \sin \theta_{\text {eff. }}$.

Since the effective polar coordinates of the $\mathrm{NH}$ vectors $\left(\theta_{\text {eff }}, \phi_{\text {eff }}\right)$ are very similar to the coordinates $(\theta, \phi)$ of the NMR structure $(1 \mathrm{~d} 3 \mathrm{z})$, this structure can be considered in a first approximation as a good dynamically averaged single-structure representation of ubiquitin.

The residue-specific $S_{\text {rdc }}^{2}$-values are shown in Figure $5 \mathrm{a}$ as derived from the individually averaged spherical harmonics according to Equation (1.4).

All secondary structure elements show reduced averaged $S_{\text {rdc }}^{2}$ values compared to the $S_{\mathrm{LS}}^{2}$ LipariSzabo order parameters, revealing large amounts of motion slower than the inverse overall tumbling correlation time of the protein. This observation is 


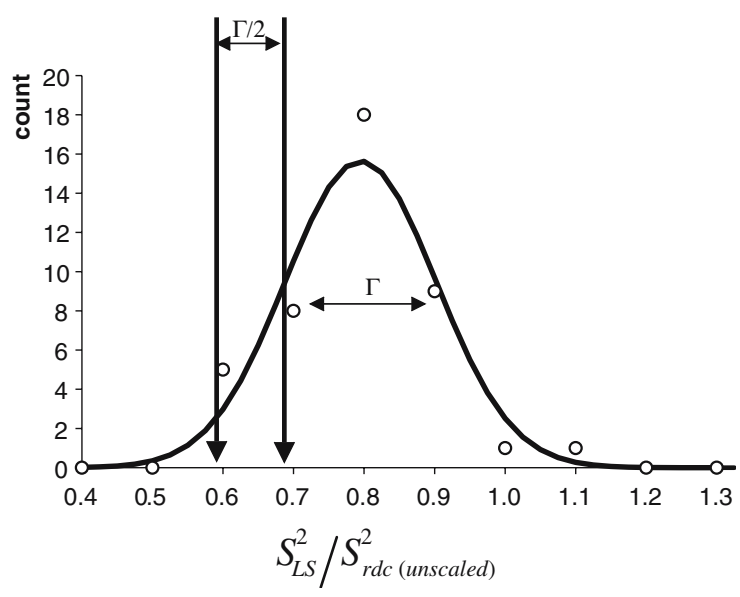

Figure 3. $S_{\text {overall }}$ is estimated utilizing the inequality $S_{\mathrm{LS}}^{2} \geq S_{\text {overall }}^{2} \cdot S_{\text {rdc(unscaled) }}^{2}$ A histogram analysis of the ratios $S_{\mathrm{LS}}^{2} / S_{\mathrm{rdc}(\text { unscaled) }}^{2}$ in ubiquitin (circles) was performed. To satisfy this inequality for all data points, $S_{\text {overall }}^{2}$ would need to be as low as 0.59 . In order to have a statistically more significant basis we added standard deviation $(\Gamma / 2=0.1)$ of the distribution of $S_{\mathrm{LS}}^{2} / S_{\mathrm{rdc}}^{2}$ (unscaled) values to this value and arrived at as a very conservative upper limit for $S_{\text {overall }}^{2}$.

most prominent for the $\beta$-strands and loop regions: the average rdc-based order parameters $S_{\text {rdc }}^{2}$ are 0.77 for the $\alpha$-helix, 0.71 for the $\beta$-sheet and 0.68 for the loop regions, compared to the average $S_{\mathrm{LS}}^{2}$ values $0.83,0.80$ and 0.78 .

For some loop regions, a higher mobility is already detected on the relaxation time scale. The decrease of order parameters is more pronounced on the $S_{\text {rdc }}^{2}$ time scale suggesting that for most loop regions the amplitudes of fast motions scale with the amplitudes of slow motion. For example, the $\mathrm{V}$-shape of $S_{\mathrm{LS}}^{2}$ values (Figure 5a) in the region spanning residues 4-16 is deepened and becomes more pronounced in the $S_{\text {rdc }}^{2}$ values.

However, for the $\alpha$-helix and $\beta$-sheet additional new modes of motion beyond the inverse overall tumbling correlation time can be detected by the rdc-based order parameters $S_{\text {rdc }}^{2}$.

While the $\alpha$-helix is rather rigid on the relaxation time scale (all $S_{\mathrm{LS}}^{2}$ are large and about the same size), rdcs detect differences between individual residues in the helix: Amino acids 23, 25, and 32 have an increased $S_{\text {rdc }}^{2}$ order parameter compared with the remaining amino acids in the $\alpha$ helix. The $\mathrm{N}$-terminal part of the $\alpha$-helix appears very rigid, while the mobility increases towards the
C-terminal part. Together with the observed directions of anisotropic motion ${\overline{\phi^{\prime}}}_{\mathrm{rdc}}$ (vide infra) this supports the idea of a slow anisotropic excursion of the helix that has also been described in an earlier publication (Meiler et al., 2003) and has recently been observed by Kithara et al. in high pressure NMR experiments (Kitahara et al., 2005).

Interestingly, the nature of the side chains seems to influence the backbone amide group mobility. Charged and polar residues reveal decreased $S_{\text {rdc }}^{2}$ order parameters compared to hydrophobic residues, indicating that backbone amide groups are more mobile for charged and polar residues. In particular, the arginines Arg42, 54,72 and 74 show very small order parameters like $0.67,0.51,0.59$ and 0.37 . These arginines are considered to play important roles in the interaction with the ubiquitin-activating enzyme E1 (Burch and Haas, 1994) and with the ubiquitinconjugating enzyme E2 (Miura et al., 1999) as well as deubiquinating enzymes (Wilkinson et al., 1999. A more detailed analysis is given by Lakomek et al., 2005).

Those amino acids that show an extremely large deviation between the $S_{\mathrm{rdc}}^{2}$ and the $S_{\mathrm{LS}}^{2}$ value are of special interest. The difference between $S_{\mathrm{LS}}^{2}$ and $S_{\text {rdc }}^{2}$ of Thr7, Asp20, Arg54, Ser65 and Val70 is greater than 0.20 , indicating that the $\mathrm{NH}$ vectors of these amino acids have large amplitude motions on a time scale slower than the correlation time. For some of these residues, one can expect motion on a time scale close to where relaxation dispersion measurements become sensitive. Indeed, for Val70 a large dispersion effect was observed at $-13^{\circ} \mathrm{C}$ (Mills and Szyperski, 2002), which corresponds to a conformational exchange rate of $133 \mu$ s. Val70 forms a hydrophobic patch together with Leu8 and Ile44 that confers specifity for the binding to different enzymes (Beal et al. 1996; Haas and Siepmann, 1997).

Whereas large motions are detected for Ile23 and Asn25 in relaxation dispersion measurements (Fushman and Cowburn, 1998; de Alba et al., 1999; Mills and Szyperski, 2002; Dittmer and Bodenhausen, 2004; Wist et al., 2004; Massi et al., 2005), both residues appear only moderately mobile in our analysis. To explain why the $S_{\text {rdc }}^{2}$ order parameter cannot detect the motion observed in the relaxation dispersion experiments, we hypothesize that the amide chemical shift of Ile23 and Asn25 is modulated either by 
(a)
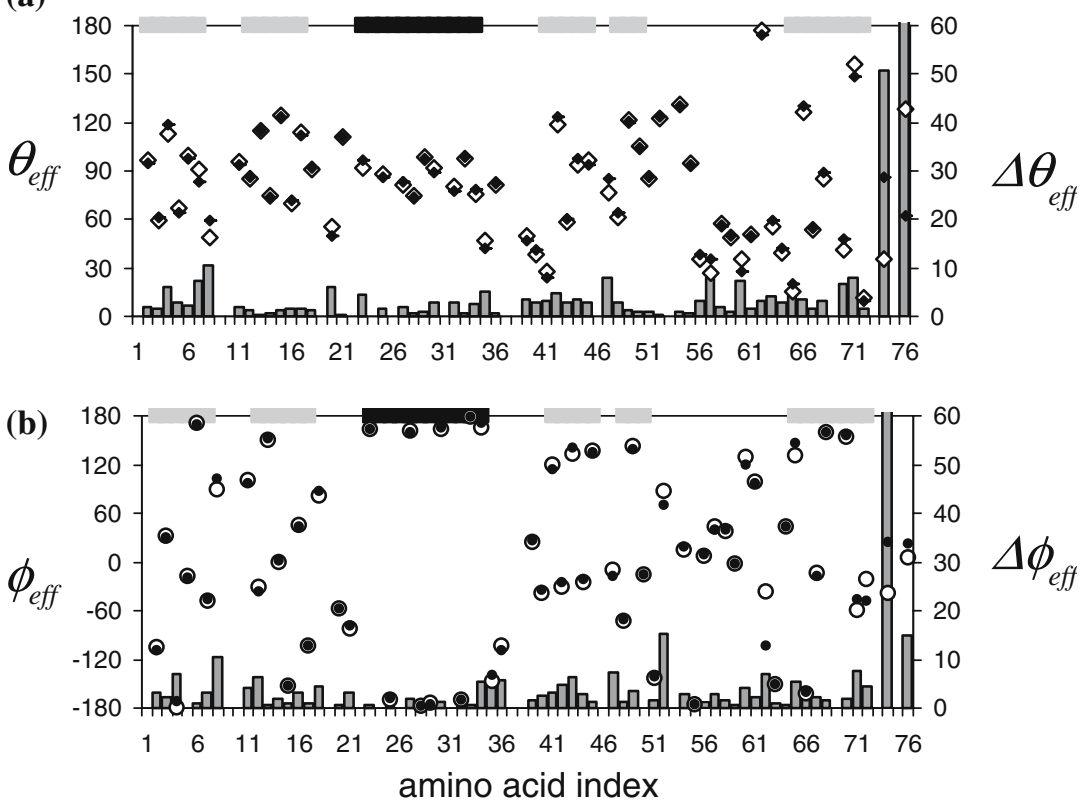

Figure 4. The effective orientation of each $\mathrm{NH}$ vector described by the polar angles $\theta_{\text {eff }}$ (a) and $\phi_{\text {eff }}$ (b) (degrees on the left $y$-axis) as derived from residual dipolar couplings (filled diamonds/circles) are compared with the ones obtained from the NMR structure 1d $3 z$ (open diamonds/circles) (Cornilescu et al., 1998). The deviations $\Delta \theta_{\text {eff }}(\mathrm{a})$ and $\Delta \phi_{\text {eff }}$ (b) between the model-free derived values and the latter ones are plotted in degrees as gray bars (right $y$-axis). The deviations of $\phi_{\text {eff }}$ are scaled with $\sin \left(\theta_{\text {eff }}\right)$ to account for the spherical distortion.

the breaking of a hydrogen bridge or by side chain reorientation. Both kinds of motion would not change the orientation of the $\mathrm{NH}$ vector and thus would not be detected by rdc measurements. Disruption of the hydrogen bond between Ile23 and Asp54 as a potential mechanism of exchange broadening for Ile23 would lead to a minor state population of $p_{\mathrm{B}} \approx 0.02$ (Massi et al., 2005) and would be undetectable by $S_{\text {rdc }}^{2}$ order parameters. However, a high amplitude of anisotropic motion $\left(\eta_{\mathrm{rdc}}=0.11\right)$ with respect to the high $S_{\mathrm{rdc}}^{2}$ value is found for Ile23, supporting the idea of a conformational exchange mechanism involving the disruption of a hydrogen bond (Massi et al., 2005).

Comparison of our $S_{\text {rdc }}^{2}$ with $S_{\mathrm{MD}}^{2}$ order parameters derived very recently from a $200 \mathrm{~ns}$ molecular dynamics simulation (Nederveen and Bonvin, 2005) reveals a good correlation (cf. Supporting information) and strongly supports our observation of slow motion beyond the overall tumbling correlation time. Both order parameters follow a similar pattern. The agreement is especially good in the $\beta$-strands. For the loop regions the MD simulation reports a strongly increased mobility that seems even more pronounced than that detected by the $S_{\text {rdc }}^{2}$ values. Whereas the $\mathrm{N}$-terminal part of the $\alpha$-helix appears quite rigid, both methods detect an increase in mobility towards the $\mathrm{C}$-terminal part of the $\alpha$-helix.

In Figure $5 b$ and $c$ the rdc-derived amplitude $\eta_{\text {rdc }}$ and direction of the asymmetry $\xi_{\text {rdc }}^{\prime}$ (with respect to the peptide plane) are presented and compared with the respective values determined from molecular dynamics. The average amplitude of asymmetry derived from rdc data is higher than the one obtained from the MD trajectory. Whereas the MD-derived directions of anisotropy have values close to $90^{\circ}$ in agreement with the $3 \mathrm{D}$ GAF model for motions of peptide planes on the Lipari-Szabo time scale (Bremi and Brueschweiler, 1997; Bremi et al., 1997; Brutscher et al., 1997; Lienin et al., 1998), larger deviations occur for the $\xi_{\text {rdc }}^{\prime}$ values indicating additional modes of motion in the ns to $\mu \mathrm{s} / \mathrm{ms}$ time scale.

\section{Anisotropic motion}

Except for the assumption of the independence of structure and dynamics from the alignment medium, the analysis presented here is "model- 

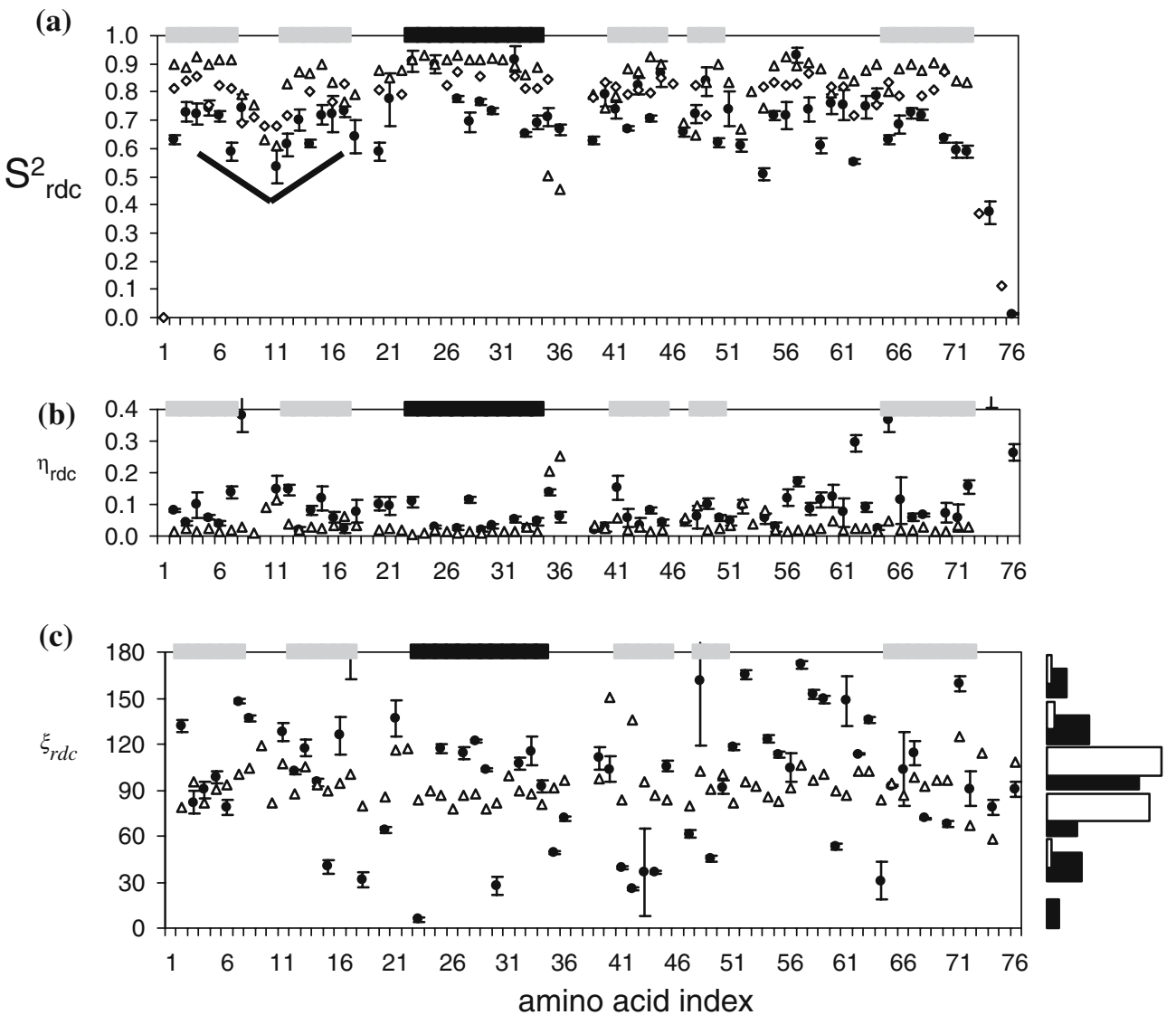

(d)

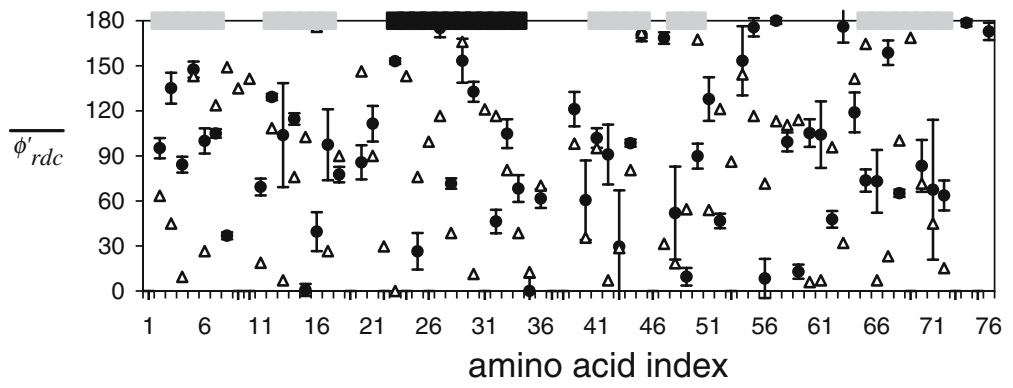

Figure 5. (a) Shows a comparison of ubiquitin order parameters obtained from 31 sets of residual dipolar couplings $S_{\text {rdc }}^{2}$ (filled circles) with the values obtained from relaxation measurements $S_{\mathrm{LS}}^{2}$ (diamonds) as well as values derived from a $10 \mathrm{~ns}$ molecular dynamics trajectory $S_{\text {traj }}^{2}$ (Meiler et al., 2001) (triangles). Helical regions in ubiquitin are marked with a black bar on top of the diagram, strand regions with a gray bar. (b) Compares the asymmetry parameter derived from residual dipolar couplings $\eta_{\text {rdc }}$ (filled circles) with values derived from a $10 \mathrm{~ns}$ molecular dynamics trajectory $\eta_{\text {traj }}$ (triangles) (Meiler et al. 2001). In (c) the $\xi_{\text {rdc }}^{\prime}$ angles as determined from experimental data (filled circles) are compared with the ones from the trajectory (triangles). The $\xi_{\text {rdc }}^{\prime}$ angle measures the direction of the asymmetry with respect to the peptide plane. In the right panel the distribution plot illustrates the strong preference for this angle to be $90^{\circ}$ in the trajectory and how this preference is weakened when looking at longer time scales utilizing experimental residual dipolar couplings. (d) Compares the $\overline{\phi^{\prime}}$ rdc angles as determined from experimental data (circles) with the ones from the trajectory (triangles). The $\overline{\phi_{\text {rdc }}^{\prime}}$ describe the direction of the anisotropic motion in a model-free manner.

free", since no models about the potential modes of motion of the NH vectors are made. Generally, asymmetries in loop regions are on average larger than in the secondary structure elements. Indeed, the average amplitude of anisotropy is $\bar{\eta}_{\text {rdc }}=0.12$ for loop regions (without C-termi- 
nus), whereas for the $\alpha$-helix and $\beta$-strands the average values are $\bar{\eta}_{\text {rdc }}=0.06$ and $\bar{\eta}_{\text {rdc }}=0.07$. Exceptionally high anisotropy was observed for the residues L8, Q62 and S65 and the residues of the $\mathrm{C}$-terminus, all of them located in loop regions.

Interestingly, the anisotropic motion of the $\alpha$-helix detected by rdcs correlates quite well with the excursion of the $\alpha$-helix observed by high pressure studies (Kitahara et al., 2005). We compared model-free derived ${\overline{\phi^{\prime}}}_{\text {rdc }}$ with the high pressure NMR results by back-calculating $\bar{\phi}_{r d c, H P}^{\prime}$ values from a two structure-ensemble of the $1 \mathrm{~V} 80$ (30 bar) and 1V81 (3 kbar) structures (cf. Supplementary material). For most of the helix residues both values deviate by less than $20^{\circ}$. Outliers are the residues 23, 30 and 33. However, the high pressure structure has been determined without the use of rdcs and therefore might contain errors on the directions of the $\mathrm{NH}$ vectors.

\section{Comparison with previous analysis}

In Figure 6a we compare the $S_{\text {rdc }}^{2}$ values published in our previous analysis (Peti et al., 2002) with the ones reported in this work. Including the additional 20 sets of data reduced the standard deviations by a factor of four. $73.8 \%$ of the data points obtained in both experiments are identical within their standard deviation, which is close to the expected percentage of $77.5 \%{ }^{1}$. The visible differences between the two sets of $S_{\text {rdc }}^{2}$ can be explained with the rather large standard deviations in our first analysis based on only 11 different alignment conditions. Furthermore, in the previous analysis, $S_{\text {overall }}$ was determined with respect to Lipari-Szabo order parameters measured at $27^{\circ} \mathrm{C}$, whereas for the current analysis newly available $S_{\mathrm{LS}}^{2}$ values at $35^{\circ} \mathrm{C}$ were used (Chang and Tjandra, 2005).

\section{Structural noise}

To evaluate the influence of structural noise (Zweckstetter and Bax, 2002) on the model-free analysis of rdcs, the rigid structure used to derive

\footnotetext{
${ }^{1}$ Statistical probability that two experimental observables of one and the same parameter are identical within their standard deviations, if the ratio between the standard deviations in the two experiments is 4:1. The standard deviation of the $S_{\mathrm{rdc}}$ in our previous experiment was 0.08 and is now reduced to 0.021 .
}

the individual alignment tensors was systematically varied. We used three different starting structures including two X-ray structures (Vijay Kumar et al., 1987; Ramage et al., 1994) and the first model of the NMR ensemble (Cornilescu et al., 1998). As the 10 models derived from NMR data $(1 \mathrm{~d} 3 \mathrm{z})$ are all very similar, only the first of the 10 models was used. The X-ray structure (PDB code: $1 \mathrm{ubq}$ ) reflects about $5 \%$ of structural noise with respect to the NMR structure (Zweckstetter and Bax, 2002). Alignment tensors differ by less than $2 \%$ in size, less than $5 \%$ in rhombicity and less than $5^{\circ}$ in the angles defining the Euler rotation of the alignment frame with respect to the molecular frame. Figure $6 \mathrm{~b}$ shows the $S_{\text {rdc }}^{2}$ (including error bars) derived when the alignment tensors were calculated for the three different structures. The obtained $S_{\text {rdc }}^{2}$ values depend only weakly on the structure that is used for determination of alignment tensors. The correlation coefficients between the three sets of $S_{\mathrm{rdc}}^{2}$-values are larger than 0.99. Structural noise has therefore only a small influence on the results of the calculation. Thus, the observed deviations of $S_{\mathrm{rdc}}^{2}$ from the $S_{\mathrm{LS}}^{2}$ order parameters cannot be accounted for by structural noise but, indeed, reveals additional motions present on a time-scale slower than the correlation time.

The $S_{\text {rdc }}^{2}$ order parameters presented in Figure $5 \mathrm{a}$ are the average values of $S_{\text {rdc }}^{2}$ that were obtained for the three different structures (1 NMR and $2 \mathrm{X}$-ray) used for the determination of alignment tensors (vide supra). The error margins reflect the standard deviation.

Recently, Clore and Schwieters pointed out that refinement of NMR structures against dipolar couplings using a single model-representation is justified for most practical applications and will not adversely affect the accuracy of the resulting coordinates. They introduced an $S_{\text {jump }}^{2}$ order parameter based on a two-site jump model which describes the minimum amount of anisotropic backbone internal motions that needs to be invoked to account for dipolar coupling data measured in multiple alignment media (Clore and Schwieters, 2004). Since this order parameter only reflects the anisotropic part of the motion, it is expected that the $S_{\text {jump }}^{2}$ order parameter is a theoretical upper limit for the rdcbased order parameter $S_{\text {rdc }}^{2}$. According to our data, however, much more motion on the slow 

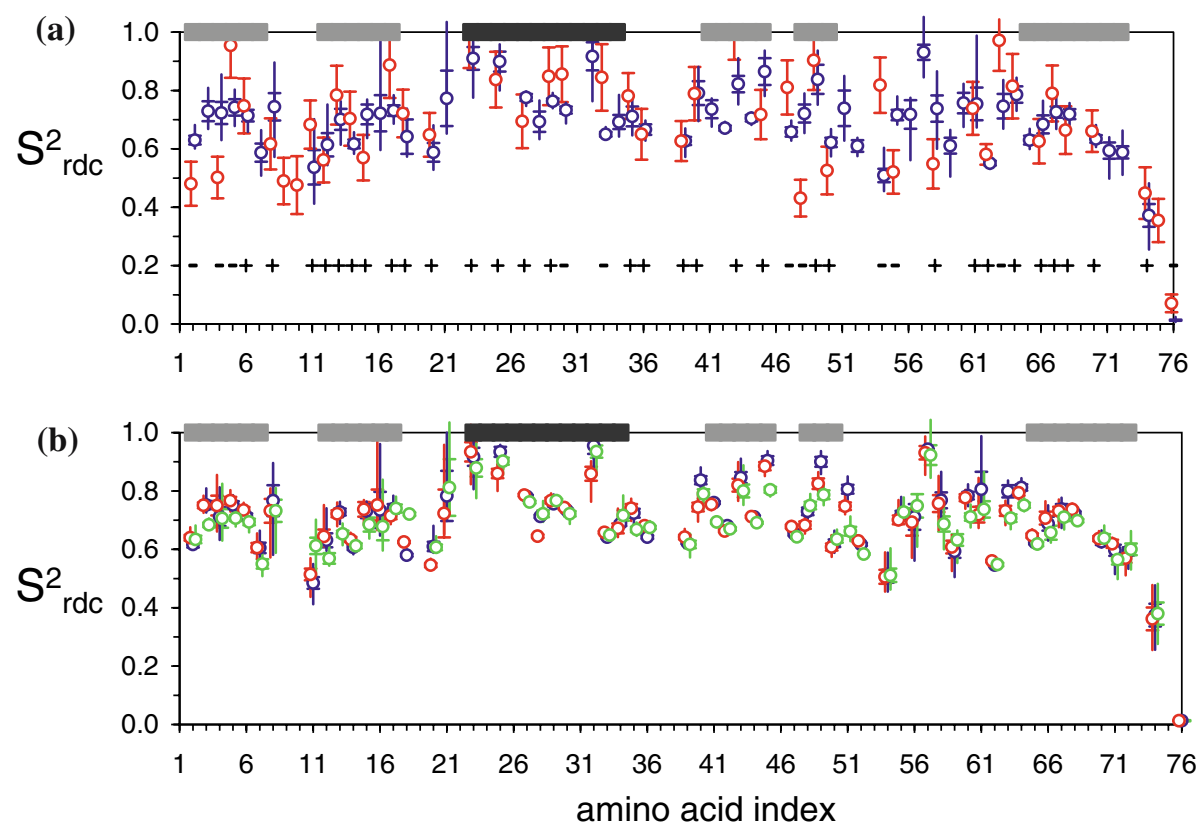

Figure 6. (a) Shows the $S_{\text {rdc }}^{2}$ values obtained from this analysis (blue circles) with the previously published data (red circles) (Peti et al., 2002). A "+" or a "-" in the lower half of the diagram indicate agreement or disagreement, respectively, within the standard deviation of our old analysis. The influence of structural noise on the analysis is evaluated in (b) by calculating the $S_{\text {rdc }}^{2}$ values using three different structures for determination of alignment tensors (1d3z - green, 1ubi - blue, and 1ubq - red). The standard and maximal deviations are marked by error bars.

time scale is present than indicated by the $S_{\text {jump }}^{2}$ order parameters.

Significance of motional anisotropy by backcalculation of residual dipolar couplings

To verify the significance of anisotropies, the NH rdcs were back-calculated under certain regimes. We replaced the averaged spherical harmonics in Equation (1.3) with the spherical harmonics in the doubly primed coordinate system, which we rotate from the doubly primed system into the molecular system by $R\left(-\phi_{\mathrm{eff}},-\theta_{\mathrm{eff}},-\overline{\phi_{\mathrm{rdc}}^{\prime}}\right)$ :

$$
\begin{aligned}
& \frac{D_{i}^{\text {exp }}}{\tilde{D}_{i, z z}} \cdot S_{\text {overall }} \stackrel{?}{=} D^{\text {backcalculated }} \\
& =\sum_{M=-2}^{2}\left[F_{i, M} \cdot \sum_{N=-2,0,2} \mathrm{e}^{-i N \phi_{i}} d_{N M}^{2}\left(\theta_{i}\right) \mathrm{e}^{-i M \overline{\phi_{i, r d}^{\prime}}}\left\langle Y_{2 N}\left(\theta^{\prime \prime}, \phi^{\prime \prime}\right)\right\rangle\right]
\end{aligned}
$$

Using this equation, the experimental rdcs were back-calculated from the obtained angles $-\phi_{\mathrm{eff}},-\theta_{\mathrm{eff}},-{\overline{\phi_{\mathrm{rdc}}^{\prime}}}_{\mathrm{f}}$ and spherical harmonics $\left\langle Y_{22}\left(\theta^{\prime \prime}, \phi^{\prime \prime}\right)\right\rangle,\left\langle Y_{2-2}\left(\theta^{\prime \prime}, \phi^{\prime \prime}\right)\right\rangle, \quad$ and $\left\langle Y_{20}\left(\theta^{\prime \prime}, \phi^{\prime \prime}\right)\right\rangle$ assuming different scenarios: (i - no dynamics) by setting $\left\langle Y_{22}\right\rangle=\left\langle Y_{2-2}\right\rangle=0$ and $\left\langle Y_{20}\right\rangle=1$, (ii axially symmetric dynamics) by setting only $\left\langle Y_{22}\right\rangle=\left\langle Y_{2-2}\right\rangle=0$, (iii - axial plus non-axially symmetric dynamics) by using $\left\langle Y_{22}\right\rangle,\left\langle Y_{2-2}\right\rangle$, and $\left\langle Y_{20}\right\rangle$ unaltered with respect to the result of our model-free analysis, and (iv - inversed asymmetry) by setting ${\overline{\phi_{\text {rdc }}^{\prime}}}^{\prime}={\overline{\phi_{\text {rdc }}^{\prime}}}^{\prime}+90^{\circ}$. The distribution of the deviation between experimental and backcalculated values in these four scenarios is shown in Figure 7. We obtain the relative deviation to the size of the alignment tensor, which we express in $\%$. The standard deviation of back-calculated and experimental deviations is $9.7 \%$, if dynamics is neglected (i). If now axially symmetric motion is assumed (ii), the agreement improves and the standard deviation drops to $4.9 \%$. By including also anisotropic motion (iii), the standard deviation drops to $2.9 \%$. The standard deviation increases to $8.4 \%$, if the asymmetry is inverted (iv). Thus, by invoking anisotropic motion, the best agreement between experimental and back- 


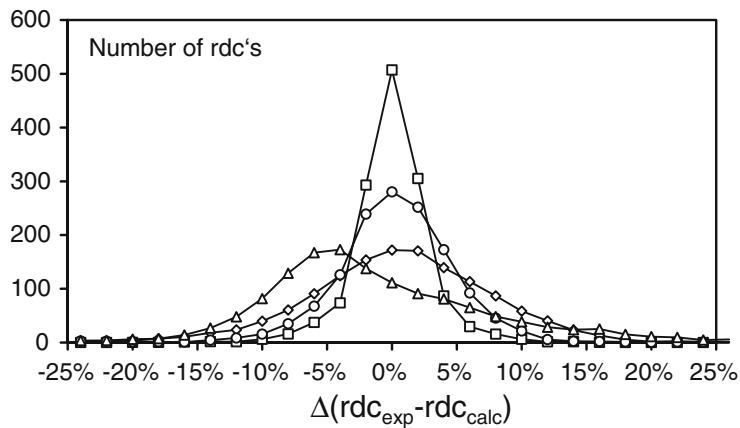

Figure 7. Agreement of the experimental data with the backcalculated rdcs assuming different models of dynamics as explained in the text: triangles - single static structure (i), circles - axial-symmetric dynamics only (ii), squares - axial-symmetric and asymmetric motion (iii), diamonds - axial-symmetric and asymmetric motion with inverted amplitudes of the asymmetry (iv). The systematic deviation seen for a single static structure result from a uniform downscaling of the experimental values due to dynamics that cannot be reflected by a static model. Usually this effect is not visualized but the alignment tensor is reduced to absorb this effect.

calculated rdcs is achieved, which strongly supports the physical relevance of anisotropic motion.

\section{Conclusions}

We performed a model-free analysis of structure and dynamics of ubiquitin using a very broad experimental data basis of NH residual dipolar couplings (rdcs) in 31 different alignment conditions. Rdc-based order parameters $S_{\mathrm{rdc}}^{2}$ were derived with so far unattained accuracy $(3 \%$ experimental error on average) revealing new modes of motion in a time window between the rotational correlation time and the low millisecond time scale. Charged and polar residues show more mobile backbone amide groups than hydrophobic residues. In particular the biologically relevant arginines reveal strongly decreased $S_{\mathrm{rdc}}^{2}$ values. Interestingly, most $S_{\mathrm{rdc}}^{2}$ values correlate quite well with order parameters derived from a 200 ns molecular dynamics simulation on ubiquitin (Nederveen and Bonvin, 2005).

Besides the $S_{\text {rdc }}^{2}$ based order parameters, we could determine effective orientations of the $\mathrm{NH}$ internuclear vectors described by the polar angles $\left(\theta_{\text {eff }}, \phi_{\text {eff }}\right)$ and the amplitudes $\eta_{\text {rdc }}$ and directions of anisotropic motions $\overline{\phi_{\text {rdc }}^{\prime}}$. The effective orientations deviate generally by less than $5 \%$ from the polar angles directly extracted from the NMR structure $(1 \mathrm{~d} 3 \mathrm{z})$, which can thus be regarded, at least in first approximation, as a good singlestructure representation of the dynamically averaged ubiquitin structure.

For the $\alpha$-helix, the determined ${\overline{\phi^{\prime}}}_{\text {rdc }}$ support an excursion of the helix as suggested earlier (Meiler et al., 2003) and recently observed by high pressure NMR (Kitahara et al., 2005). The influence of structural noise on the model free analysis has been shown to be negligible.

Supplementary material to this paper is available in electronic format at http://dx.doi.org/ $10.1007 / \mathrm{s} 10858-005-5686-0$.

\section{Acknowledgements}

This work was supported by the MPG, the DFG and the Fonds der Chemischen Industrie (to CG). We thank Dirk Lennartz (Bayer Leverkusen) and Vinesh Vijayan (MPI Goettingen) for help with the measurement of dipolar couplings, as well as Wolfgang Peti (Brown University), Jeanine Prompers (University of Nijmegen) and Rafael Brüschweiler (Florida State University) for continuous discussion and interest. Furthermore, we would like to thank Nico Tjandra (NIH) for providing the Lipari-Szabo order parameters and Marius Clore (NIH) as well as Joel R. Tolman (Johns Hopkins University) for interesting and sometimes controversial discussions on the subject. We thank Karin Giller for expert technical help expressing the ubiquitin. J.M. thanks the Human Frontier Science Program (HFSP) for financial support.

\section{References}

Akke, M. and Palmer, A.G. (1996) J. Am. Chem. Soc., 118, 911-912.

Barrientos, L.G., Dolan, C. and Gronenborn, A.M. (2000) J. Biomol. NMR, 16, 329-337.

Beal, R., Deveraux, Q., Xia, G., Rechsteiner, M. and Pickart, C. (1996) Proc. Natl. Acad. Sci. U.S.A, 93, 861-866.

Bernado, P. and Blackledge, M. (2004a) J. Am. Chem. Soc., 126, 4907-4920.

Bernado, P. and Blackledge, M. (2004b) J. Am. Chem. Soc., 126, 7760-7761.

Bertoncini, C.W., Jung, Y.S., Fernandez, C.O., Hoyer, W., Griesinger, C., Jovin, T.M. and Zweckstetter, M. (2005) Proc. Natl. Acad. Sci. U.S.A, 102, 1430-1435.

Bremi, T. and Brueschweiler, R. (1997) J. Am. Chem. Soc., 119, 6672-6673. 
Bremi, T., Brueschweiler, R. and Ernst, R.R. (1997) J. Am. Chem. Soc., 119, 4272-4284.

Briggman, K.B. and Tolman, J.R. (2003) J. Am. Chem. Soc., 125, 10164-10165.

Brutscher, B., Brueschweiler, R. and Ernst, R.R. (1997) Biochemistry, 36, 13043-13053.

Burch, T.J. and Haas, A.L. (1994) Biochemistry, 33, 73007308.

Chang, S.-L. and Tjandra, N. (2005) J. Magn. Res., 174, 43-53.

Chou, J.J., Gaemers, S., Howder, B., Louis, J.M. and Bax, A. (2001) J. Biomol. NMR, 21, 377-382.

Clore, G.M. and Schwieters, C.D. (2004) J. Am. Chem. Soc., 126, 2923-2938.

Cornilescu, G., Marquardt, J.L., Ottiger, M. and Bax, A. (1998) J. Am. Chem. Soc., 120, 6836-6837.

de Alba, E., Baber, J.L. and Tjandra, N. (1999) J. Am. Chem. Soc., 121, 4282-4283.

Delaglio, F., Grzesiek, S., Vuister, G.W., Zhu, G., Pfeifer, J. and Bax, A. (1995) J. Biomol. NMR, 6, 277-293.

Dittmer, J. and Bodenhausen, G. (2004) J. Am. Chem. Soc., 126, 1314-1315.

Fushman, D. and Cowburn, D. (1998) J. Am. Chem. Soc., 120, 7109-7110.

Haas, A.L. and Siepmann, T.J. (1997) FASEB J., 11, 12571268.

Johnson, E.C., Lazar, G.A., Desjarlais, J.R. and Handel, T.M. (1999) Structure Fold Des., 7, 967-976.

Kay, L.E., Torchia, D.A. and Bax, A. (1989) Biochemistry, 28, 8972-8979.

Kitahara, R., Yokoyama, S. and Akasaka, K. (2005) J. Mol. Biol., 347, 277-285.

Koenig, B.W., Hu, J.-S., Ottiger, M., Bose, S., Hendler, R.W. and Bax, A. (1999) J. Am. Chem. Soc., 121, 1385-1386.

Kopple, K.D., Bhandary, K.K., Kartha, G., Wang, Y.S. and Parameswaran, K.N. (1986) J. Am. Chem. Soc., 108, 46374642 .

Lakomek, N.A., Farés, C., Becker, S., Carlomagno, T., Meiler, J. and Griesinger, C. (2005) Angew. Chem. Int. Ed., 44, 77767778 .

Lienin, S.F., Bremi, T., Brutscher, B., Brueschweiler, R. and Ernst, R.R. (1998) J. Am. Chem. Soc., 120, 9870-9879.

Lindorff-Larsen, K., Best, R.B., Depristo, M.A., Dobson, C.M. and Vendruscolo, M. (2005) Nature, 433, 128-132.

Lipari, G. and Szabo, A. (1982a) J. Am. Chem. Soc., 104, 45464559.

Lipari, G. and Szabo, A. (1982b) J. Am. Chem. Soc., 104, 45594570.

Losonczi, J.A. and Prestegard, J.H. (1998) J. Biomol. NMR, 12, 447-451.

Massi, F., Grey, M.J. and Palmer, A.G. (2005) Protein Sci., 14, 735-742.

Meiler, J., Peti, W. and Griesinger, C. (2000) J. Biomol. NMR, 17, 283-294.
Meiler, J., Peti, W., Prompers, J., Griesinger, C. and Brueschweiler, R. (2001) J. Am. Chem. Soc., 123, 6098-6107.

Meiler, J., Peti, W. and Griesinger, C. (2003) J. Am. Chem. Soc., 125, 8072-8073.

Mills, J.L. and Szyperski, T. (2002) J. Biomol. NMR, 23, 63-67.

Miura, T., Klaus, W., Gsell, B., Miyamoto, C. and Senn, H. (1999) J. Mol. Biol., 290, 213-228.

Nederveen, A.J. and Bonvin, A.M.J.J. (2005) J. Chem. Theory Comput., 1, 363-374.

Ottiger, M., Delaglio, F. and Bax, A. (1998) J. Magn. Res., 131 373-378.

Palmer, A.G. 3rd (2004) Chem. Rev., 104, 3623-3640.

Peti, W., Meiler, J., Brueschweiler, R. and Griesinger, C. (2002) J. Am. Chem. Soc., 124, 5822-5833.

Ramage, R., Green, J., Muir, T.W., Ogunjobi, O.M., Love, S. and Shaw, K. (1994) Biochem. J., 299, 151-158.

Ruckert, M. and Otting, G. (2000) J. Am. Chem. Soc., 122, 7793-7797.

Sass, H.J., Musco, G., Stahl, S.J., Wingfield, P.T. and Grzesiek, S. (2000) J. Biomol. NMR, 18, 303-309.

Schneider, D.M., Dellwo, M.J. and Wand, A.J. (1992) Biochemistry, 31, 3645-3652.

Sivaraman, T., Arrington, C.B. and Robertson, A.D. (2001) Nat. Struct. Biol., 8, 331-333.

Tjandra, N., Feller, S.E., Pastor, R.W. and Bax, A. (1995) J. Am. Chem. Soc. 117, 12562-12566.

Tolman, J.R. (2001) Curr. Opin. Struct. Biol., 11, 532-539.

Tolman, J.R. (2002) J. Am. Chem. Soc., 124, 12020-12030.

Tolman, J.R., Flanagan, J.M., Kennedy, M.A. and Prestegard, J.H. (1995) Proc. Natl. Acad. Sci. U.S.A, 92, 9279-9283.

Tolman, J.R. and Prestegard, J.H. (1996a) J. Magn. Reson. B, 112, 245-252.

Tolman, J.R. and Prestegard, J.H. (1996b) J. Magn. Reson. B, 112, 269-274.

Tolman, J.R., Flanagan, J.M., Kennedy, M.A. and Prestegard, J.H. (1997) Nat. Struct. Biol., 4, 292-297.

Tolman, J.R., Al-Hashimi, H.M., Kay, L.E. and Prestegard, J.H. (2001) J. Am. Chem. Soc., 123, 1416-1424.

Tycko, R., Blanco, F.J. and Ishii, Y. (2000) J. Am. Chem. Soc., 122, 9340-9341.

Vijay Kumar, S., Bugg, C.E. and Cook, W.J. (1987) J. Mol. Biol., 194, 531-544.

Wilkinson, K.D., Laleli-Sahin, E., Urbauer, J., Larsen, C.N., Shih, G.H., Haas, A.L., Walsh, S.T. and Wand, A.J. (1999) J. Mol. Biol., 291, 1067-1077.

Wist, J., Frueh, D., Tolman, J.R. and Bodenhausen, G. (2004) J. Biomol. NMR, 28, 263-272.

Zweckstetter, M. and Bax, A. (2001) J. Biomol. NMR, 20, 365 377.

Zweckstetter, M. and Bax, A. (2002) J. Biomol. NMR, 23, 127-137. 